\title{
Carotid Baroreceptor Stimulation for the Treatment of Resistant Hypertension
}

\author{
Vasilios Papademetriou, ${ }^{1}$ Michael Doumas, ${ }^{2}$ Charles Faselis, ${ }^{2}$ Constantinos Tsioufis, ${ }^{1}$ \\ Stella Douma, ${ }^{3}$ Eugene Gkaliagkousi, ${ }^{3}$ and Chrysanthos Zamboulis ${ }^{3}$ \\ ${ }^{1}$ Veterans Affairs Medical Center, Georgetown University, Washington, DC 20422, USA \\ ${ }^{2}$ Veterans Affairs Medical Center, George Washington University, Washington, DC 20037, USA \\ ${ }^{3}$ 2nd Propedeutic Department of Internal Medicine, Aristotle University of Thessaloniki, 54643 Thessaloniki, Greece
}

Correspondence should be addressed to Michael Doumas, michalisdoumas@yahoo.co.uk

Received 2 October 2010; Accepted 28 February 2011

Academic Editor: Henry Punzi

Copyright (C) 2011 Vasilios Papademetriou et al. This is an open access article distributed under the Creative Commons Attribution License, which permits unrestricted use, distribution, and reproduction in any medium, provided the original work is properly cited.

\begin{abstract}
Interventional activation of the carotid baroreflex has been an appealing idea for the management of resistant hypertension for several decades, yet its clinical application remained elusive and a goal for the future. It is only recently that the profound understanding of the complex anatomy and pathophysiology of the circuit, combined with the accumulation of relevant experimental and clinical data both in animals and in humans, has allowed the development of a more effective and wellpromising approach. Indeed, current data support a sustained over a transient reduction of blood pressure through the resetting of baroreceptors, and technical deficits have been minimized with a subsequent recession of adverse events. In addition, clinical outcomes from the application of a new implantable device (Rheos) that induces carotid baroreceptor stimulation point towards a safe and effective blood pressure reduction, but longer experience is needed before its integration in the everyday clinical practice. While accumulating evidence indicates that carotid baroreceptor stimulation exerts its benefits beyond blood pressure reduction, further research is necessary to assess the spectrum of beneficial effects and evaluate potential hazards, before the extraction of secure conclusions.
\end{abstract}

\section{Introduction}

Arterial hypertension represents a major public health problem around the word. Currently, more than one billion people are thought to have hypertension worldwide and the number is estimated to exceed 1.5 billion by 2025 [1]. The advent of antihypertensive therapy has provided drugs that effectively lower blood pressure and contributed significantly to the reduction of cardiovascular events [2]. The rates of awareness, treatment, and control of hypertension have been constantly increased over the last decades; they remain, however, far from optimal [3], underlining the need for the implementation of more effective approaches.

Antihypertensive drugs belong to different categories, exerting their actions through different mechanisms that are sometimes complimentary. It has been shown, however, that despite the proper use of several antihypertensive agents, blood pressure remains uncontrolled in a small percentage of hypertensive patients (5\%-15\%). This subgroup has been called over the years as suffering from refractory, difficult to control, or resistant hypertension (lately). Although the percentage of this subgroup does not seem significant, the actual number of resistant hypertensives is estimated to be very large due to the high prevalence of hypertension in the general population. Therefore, the need for alternative approaches has been widely recognized over the last several years. This is the reason why the interventional management of hypertension, which has been used at the mid of the 20th century and abandoned thereafter, has rekindled and gained intense scientific interest. In particular, carotid baroreceptor stimulation and renal sympathetic denervation have been tested during the last decade for the treatment of resistant hypertension with promising preliminary results. 
The carotid baroreflex represents a significant element of blood pressure homeostasis. Carotid baroreceptors sense the intra-arterial blood pressure and modulate the sympathetic tone towards the opposite direction that is, high blood pressure results in reduced sympathetic tone through baroreceptor activation, while enhanced sympathetic tone compensates for low blood pressure. For a long period of time, the carotid baroreflex has been considered as a shortterm buffering system, regulating the abrupt transient fluctuations of blood pressure around a "constant set-point", while its role in the long-term regulation of blood pressure has been significantly questioned. Recent experimental and clinical data however challenge this long-standing belief, and strongly suggest the ability of the carotid baroreflex to exert long-term effects on blood pressure; therefore, the interventional activation of the carotid baroreflex has revived for the management of resistant hypertension.

This paper aims to present available data on the role of the carotid baroreflex in the treatment of resistant hypertension by: (a) delineating the anatomy, physiology, and pathophysiology of the baroreflex arc, (b) presenting the experience with carotid nerve activation that was used in the 60 s for the treatment of severe hypertension and other cardiovascular diseases, and (c) by critically evaluating recent experimental and clinical data with carotid baroreceptor stimulation using the Rheos device.

\section{Anatomy, Physiology, and Pathophysiology of the Carotid Baroreflex}

The carotid baroreflex circuit represents the main part of the arterial baroreflex system. Although peripheral baroreceptors can be found in the aortic arch, the heart, and the pulmonary vessels, a vast amount of evidence points towards the pivotal role of the carotid baroreceptors on blood pressure buffering. Carotid baroreceptors are stretch-sensitive mechanosensors, located at the right and left carotid sinus. They sense the distention of the carotid wall and transmit signals to the brain stem via the glossopharyngeal nerve. The nucleus tractus solitaris (NTS) that lies in the dorsal medulla represents the "reception center" of afferent signals from arterial baroreceptors. Signals are then neurotransmitted to the caudal ventrolateral medulla (CVLM), which represents the "conversion center", since it converts the excitatory signals from peripheral baroreceptors to inhibitory signals that sequentially travel to the rostral ventrolateral medulla (RVLM). The latter represents the "coordinating center" since sympathoexcitatory neurons travel from here towards all over the body regulating the sympathetic tone.

Actually, the baroreflex circuit is much more complicated than the afore-mentioned brief description. Anatomical crossovers, intersections, and bypasses exist, while a great variety of peptides are used as neurotransmitters with either inhibiting or activating properties. Moreover, data from anatomical, pharmacological, and electrophysiological studies are sometimes conflicting, mainly due to the sophisticated and demanding techniques that are used for these studies, thus rendering methodological problems unavoidable.
However, a detailed description of the carotid baroreflex is beyond the scope of the current paper. Therefore, it can be summarized that, irrespective of the exact nature of the baroreflex circuit, the net result of baroreceptor activation is a subsequent suppression of the sympathetic tone.

The functional evaluation of the carotid baroreflex has been mainly based on suppression studies through carotid denervation. A large amount of animal studies with carotid denervation in different species provided essential information regarding the role of carotid baroreceptors in the regulation of blood pressure, especially during the long term. Older studies have mainly pointed towards a transient increase of blood pressure following baroreceptor denervation that was not sustained during longer followup periods, despite some opposite findings that have been reported [4-10]. A limited role of carotid baroreflex in the long-term regulation of blood pressure was further established by the landmark study of McCubbin, which described the phenomenon of "baroreceptor resetting" [11]. The response to the carotid baroreflex is blunted in hypertensive animals suggesting a resetting of the carotid baroreceptors towards the prevailing pressure, which is actually very rapid and takes place within minutes [12]. However, several concerns can be raised regarding the denervation studies as well as the level of baroreceptor resetting that have been recently reviewed [13]. Indeed, recent animal studies suggest a significant role of the carotid baroreflex even in the long-term blood pressure regulation, by using different, more sophisticated, methodological approaches that better resemble clinical conditions [14-20].

Data in humans exist as well, although limited, and derive from denervation studies reporting the effects of iatrogenic damage of carotid sinus during various procedures (carotid body tumour surgery, carotid paraganglioma excision, carotid endarterectomy, and head and neck radiotherapy). The majority of these studies failed to find a persistent elevation of blood pressure, despite acute transient increases of blood pressure levels and enhanced blood pressure variability [21-25]; however, opposite studies can be found as well, reporting long-lasting blood pressure elevations following carotid baroreceptor denervation [26-28]. The above-mentioned controversial findings may be attributed to the retrospective nature of the studies that has not permitted for appropriate study designs.

\section{Carotid Nerve Activation-Experimental and Clinical Data}

Carotid baroreflex activation for the treatment of resistant hypertension does not represent a new idea. The pursuit of blood pressure reduction through the continuous baroreflex activation has flourished during the 50s and 60s. Several devices have been invented for the exogenous electrical stimulation of the carotid nerves. The principal idea was that an external generator transmitted signals to an implanted receiver through an antenna coil, and the signals subsequently travelled to electrodes that were placed in contact with the carotid sinus nerves. The concept was based on 
the assumption that the continuous carotid nerve signalling would be sensed by the central nervous system as a constant rise in blood pressure, leading to sympathetic attenuation and subsequent blood pressure reduction. This concept created a lot of enthusiasm, and initial experimental studies have been shortly thereafter followed by clinical studies. This is not surprising, given the limited armamentarium for the therapeutic management of severe hypertension at that time.

Animal studies with carotid nerve activation uncovered significant blood pressure reduction, which was not only transient but was maintained during the one-year followup study period $[29,30]$. The observation that electrical carotid nerve activation acutely decreases blood pressure in humans [31] encouraged the application of these devices in patients with resistant hypertension. Several case series from specialized clinics in North America have been published during the 60s [32-37]. The results showed a consistent blood pressure reduction in the majority of patients that was evident rapidly and lasted during prolonged followup periods, up to twelve years. The enthusiasm regarding this efficacious method however subsided rather rapidly, due to the excessive incidence of adverse events and the severe technical disadvantages of the devices.

\section{Carotid Baroreceptor Stimulation- Experimental and Clinical Data}

Recent advances in technology seem to overcome many of the prior technological problems with the devices, thus rekindling the scientific interest and rendering carotid baroreflex activation an appealing field of research. A small, USbased, pharmaceutical company (CVRx Inc, Minneapolis, Minn) has developed an implantable device (Rheos) for the electrical stimulation of carotid baroreceptors. In brief, a pulse generator that resembles a pacemaker is implanted in the right infraclavicular space and connects to two electrode leads that are placed in the perivascular space of the two carotid sinuses (Figure 1). The generator communicates with an external computer system that is capable of programming the baroreceptor activation in a noninvasive way. The procedure requires an experienced team of surgeons, anaesthesiologists, hypertension specialists, and technicians for the proper placement of the electrodes, the efficacy testing, and the overall success. The properties of the Rheos device along with the advancements in the surgical and anesthesiology field seem to address a lot of previous concerns; adverse events are not encountered as frequently as with the old devices and are mostly of less severity.

The Rheos device has been extensively tested in animals, reflecting current stringent requirements by regulatory authorities worldwide. Carotid baroreceptor stimulation was effective in reducing blood pressure of conscious normotensive dogs [38] and in obesity-induced hypertension [39]. On the other hand, blood pressure reduction was significantly attenuated, but not abolished, in angiotensin II-induced hypertension [40], suggesting that the overactivation of the renin-angiotensin system may partially overcome the effects of the carotid baroreceptor stimulation. The clinical

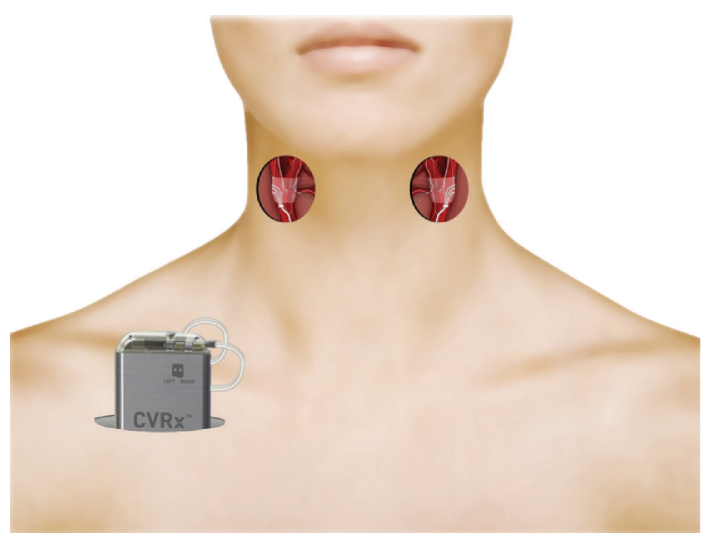

FIGURE 1: Schematic representation of the Rheos device for carotid baroreceptor stimulation.

significance of this finding and the answer to the subsequent question, whether drugs inhibiting the renin-angiotensin axis are essential in patients undergoing carotid baroreceptor stimulation for the maintenance of blood pressure reduction, remain to be clarified. Another puzzling finding comes from the study of carotid baroreceptor stimulation before and after bilateral renal denervation [41]. Blood pressure reduction with the Rheos device was unaltered by renal denervation, casting doubts on the role of renal innervation in mediating the effects of the carotid baroreflex on blood pressure regulation. However, further studies in hypertensive animals need to be performed to uncover the role of renal denervation in this experimental setting. It has to be noted, however, that in all the afore-mentioned animal studies [38-41], the blood pressure reduction was accompanied by a significant decrease in plasma noradrenaline levels, indicating that the suppression of the sympathetic tone mediates the effects of carotid baroreceptor stimulation on blood pressure.

Studies in humans have confirmed the efficacy of this interventional approach, which was observed in animals. Acute blood pressure reduction was noted by using the Rheos device during elective carotid surgery [42]. Several case reports in patients with resistant hypertension have shown the clinical utility and long-lasting reductions in blood pressure with carotid baroreceptor stimulation, setting the basis for proof-of-concept, properly designed, clinical trials [43-45]. The device based therapy of hypertension (DEBuTHT) trial in 45 patients with resistant hypertension revealed a significant reduction in both systolic and diastolic blood pressure, which was evident from the beginning of the study and was maintained thereafter [46]. The 3-year efficacy was recently presented verifying the long-lasting effects of carotid baropacing. Recruitment for a large randomized study has been completed and results are still pending. Preliminary information suggests that some patients may not respond as well, and a more careful selection process may need to be implemented.

Available data suggest a beneficial effect of carotid baroreceptor stimulation on the reversal of left ventricular hypertrophy. Moreover, additional desirable effects on cardiac 
structure and function have been observed, including attenuated mitral A-valve velocity and reduced left atrial dimensions. Whether these pilot findings would be of major clinical importance remain to be further investigated. Another important aspect relates to the effects of carotid baroreceptor stimulation on renal function. Available data suggests that carotid baropacing does not impair the renal function of patients with resistant hypertension, even during prolonged followup periods. Two recent studies enlighten the mechanisms via which carotid baroreceptor stimulation achieves blood pressure reduction $[47,48]$. Similar to findings from animal studies, it was shown that baroreceptor stimulation was accompanied by attenuation of sympathetic activity, assessed by muscle sympathetic nerve activity [47] or by analysis of heart rate variability [48]. Regarding safety, recent data indicate that the Rheos device can be safely used in patients with pacemakers [49]. In addition, it has been shown that the chronic stimulation of the carotid baroreceptors does not cause injury, remodelling, or stenosis of the carotid arteries [50].

It has to be noted that some technical issues need to be resolved, and several clinical aspects need to be clarified, before the wide application of this interventional technique in everyday clinical practice [13]. However, the latest concerns regarding the efficacy of the device underline the need for careful evaluation of every emerging therapeutic approach to fulfill the two fundamental requirements: safety and efficacy.

\section{Conclusions}

The carotid baroreflex represents an essential component of blood pressure regulation. The activation of the carotid baroreflex results in the attenuation of the sympathetic tone and subsequent blood pressure reduction. Carotid nerve activation has been used in the past for the treatment of severe hypertension, but its use has been abandoned due to adverse events and several technical disadvantages. Recent technological advances have permitted the development of a new device (Rheos) that electrically stimulates carotid baroreceptors and seems to overcome prior technical problems. Available experimental and clinical data point towards adequate efficacy with acceptable safety of this device although some concerns have been raised lately. Therefore, further studies are needed to clarify the place of carotid baroreceptor stimulation in the management of patients with resistant hypertension.

\section{References}

[1] P. M. Kearney, M. Whelton, K. Reynolds, P. Muntner, P. K. Whelton, and J. He, "Global burden of hypertension: analysis of worldwide data," Lancet, vol. 365, no. 9455, pp. 217-223, 2005.

[2] S. Lewington, R. Clarke, N. Qizilbash, R. Peto, and R. Collins, "Age-specific relevance of usual blood pressure to vascular mortality: a meta-analysis of individual data for one million adults in 61 prospective studies," Lancet, vol. 360, no. 9349, pp. 1903-1913, 2002.
[3] B. M. Egan, Y. Zhao, and R. N. Axon, "US trends in prevalence, awareness, treatment, and control of hypertension, 19882008," Journal of the American Medical Association, vol. 303, no. 20, pp. 2043-2050, 2010.

[4] M. Saito, N. Terui, Y. Numao, and M. Kumada, "Absence of sustained hypertension in sinoaortic-denervated rabbits," American Journal of Physiology, vol. 251, no. 4, pp. H742H747, 1986.

[5] C. S. Ito and A. M. Scher, "Regulation of arterial blood pressure by aortic baroreceptors in the unanesthetized dog," Circulation Research, vol. 42, no. 2, pp. 230-236, 1978.

[6] R. E. Shade, V. S. Bishop, J. R. Haywood, and C. K. Hamm, "Cardiovascular and neuroendocrine responses to baroreceptor denervation in baboons," American Journal of Physiology, vol. 258, no. 4, pp. R930-R938, 1990.

[7] V. S. Bishop, J. R. Haywood, R. E. Shade et al., "Aortic baroreceptor deafferentation in the baboon," Journal of Applied Physiology, vol. 60, no. 3, pp. 798-801, 1986.

[8] A. W. Cowley Jr., J. F. Liard, and A. C. Guyton, "Role of baroreceptor reflex in daily control of arterial blood pressure and other variables in dogs," Circulation Research, vol. 32, no. 5, pp. 564-576, 1973.

[9] C. S. Ito and A. M. Scher, "Hypertension following arterial baroreceptor denervation in the unanesthetized dog," Circulation Research, vol. 48, no. 4, pp. 576-591, 1981.

[10] P. Persson, H. Ehmke, H. Kirchheim, and H. Seller, "Effect of sino-aortic denervation in comparison to cardiopulmonary deafferentiation on long-term blood pressure in conscious dogs," Pflugers Archiv, vol. 411, no. 2, pp. 160-166, 1988.

[11] J. W. McCubbin, J. H. Green, and I. H. Page, "Baroreceptor function in chronic renal hypertension," Circulation Research, vol. 4, pp. 205-210, 1956.

[12] M. W. Chapleau, G. Hajduczok, and F. M. Abboud, "Mechanisms of resetting of arterial baroreceptors: an overview," American Journal of the Medical Sciences, vol. 295, no. 4, pp. 327-334, 1988.

[13] M. Doumas, D. Guo, and V. Papademetriou, "Carotid baroreceptor stimulation as a therapeutic target in hypertension and other cardiovascular conditions," Expert Opinion on Therapeutic Targets, vol. 13, no. 4, pp. 413-425, 2009.

[14] T. N. Thrasher, "Unloading arterial baroreceptors causes neurogenic hypertension," American Journal of Physiology, vol. 282, no. 4, pp. R1044-R1053, 2002.

[15] J. W. Osborn and B. J. Hornfeldt, "Arterial baroreceptor denervation impairs long-term regulation of arterial pressure during dietary salt loading," American Journal of Physiology, vol. 275, no. 5, pp. H1558-H1566, 1998.

[16] C. J. Barrett, R. Ramchandra, S. J. Guild, A. Lala, D. M. Budgett, and S. C. Malpas, "What sets the long-term level of renal sympathetic nerve activity: a role for angiotensin II and baroreflexes?" Circulation Research, vol. 92, no. 12, pp. 13301336, 2003.

[17] T. E. Lohmeier, J. R. Lohmeier, A. Haque, and D. A. Hildebrandt, "Baroreflexes prevent neurally induced sodium retention in angiotensin hypertension," American Journal of Physiology, vol. 279, no. 4, pp. R1437-R1448, 2000.

[18] T. N. Thrasher, "Effects of chronic baroreceptor unloading on blood pressure in the dog," American Journal of Physiology, vol. 288, no. 4, pp. R863-R871, 2005.

[19] M. W. Chapleau, C. M. Heesch, and F. M. Abboud, "Prevention or attenuation of baroreceptor resetting by pulsatility during elevated pressure," Hypertension, vol. 9, no. 6, pp. III137-III-141, 1987. 
[20] V. L. Brooks and A. F. Sved, "Pressure to change? Re-evaluating the role of baroreceptors in the long-term control of arterial pressure," American Journal of Physiology, vol. 288, no. 4, pp. R815-R818, 2005.

[21] P. Bucy, "The carotid sinus nerve in man," Archives of Internal Medicine, vol. 58, pp. 418-432, 1936.

[22] F. R. Ford, "Fatal hypertensive crisis following denervation of the carotid sinus for the relief of repeated attacks of syncope," Bulletin of the Johns Hopkins Hospital, vol. 100, pp. 14-16, 1957.

[23] D. Robertson, A. S. Hollister, I. Biaggioni, J. L. Netterville, R. Mosqueda-Garcia, and R. M. Robertson, "The diagnosis and treatment of baroreflex failure," New England Journal of Medicine, vol. 329, no. 20, pp. 1449-1455, 1993.

[24] G. De Toma, V. Nicolanti, M. Plocco et al., "Baroreflex failure syndrome after bilateral excision of carotid body tumors: an underestimated problem," Journal of Vascular Surgery, vol. 31, no. 4, pp. 806-810, 2000.

[25] H. J. L. M. Timmers, J. M. Karemaker, W. Wieling et al., "Arterial baroreflex and peripheral chemoreflex function after radiotherapy for laryngeal or pharyngeal cancer," International Journal of Radiation, Oncology, Biology, Physics, vol. 53, no. 5, pp. 1203-1210, 2002.

[26] P. Holton and J. B. Wood, "The effects of bilateral removal of the carotid bodies and denervation of the carotid sinuses in two human subjects," Journal of Physiology, vol. 181, no. 2, pp. 365-378, 1965.

[27] A. A. J. Smit, H. J. L. M. Timmers, W. Wieling et al., "Longterm effects of carotid sinus denervation on arterial blood pressure in humans," Circulation, vol. 105, no. 11, pp. 13291335, 2002.

[28] Y. Sharabi, R. Dendi, C. Holmes, and D. S. Goldstein, "Baroreflex failure as a late sequela of neck irradiation," Hypertension, vol. 42, no. 1, pp. 110-116, 2003.

[29] H. R. Warner, "The frequency-dependent nature of blood pressure regulation by the carotid sinus studied with an electric analog," Circulation Research, vol. 6, no. 1, pp. 35-40, 1958.

[30] S. I. Schwartz, L. S. C. Griffith, A. Neistadt, and N. Hagfors, "Chronic carotid sinus nerve stimulation in the treatment of essential hypertension," The American Journal of Surgery, vol. 114, no. 1, pp. 5-15, 1967.

[31] A. Carlsten, B. Folkow, G. Grimby, C. A. Hamberger, and O. Thulesius, "Cardiovascular effects of direct stimulation of the carotid sinus nerve in man," Acta Physiologica Scandinavica, vol. 44, no. 2, pp. 138-145, 1958.

[32] J. Tuckman, T. Reich, A. F. Lyon et al., "Electrical stimulation of the sinus nerves in hypertensive patients," in Hypertension, vol. 16, pp. 23-38, American Heart Association, New York, NY, USA, 3rd edition, 1968.

[33] A. N. Brest, L. Wiener, and B. Bachrach, "Bilateral carotid sinus nerve stimulation in the treatment of hypertension," The American Journal of Cardiology, vol. 29, no. 6, pp. 821-825, 1972.

[34] A. M. Bilgutay and C. W. Lillehei, "Surgical treatment of hypertension with reference to baropacing," The American Journal of Cardiology, vol. 17, no. 5, pp. 663-667, 1966.

[35] S. I. Schwartz, "Clinical applications of carotid sinus nerve stimulation," Cardiovascular Clinic, vol. 1, supplement 3, pp. 208-222, 1969.

[36] T. K. Peters, H. E. Koralewski, and E. Zerbst, "Search for optimal frequencies and amplitudes of therapeutic electrical carotid sinus nerve stimulation by application of the evolution strategy," Artificial Organs, vol. 13, no. 2, pp. 133-143, 1989.
[37] T. K. Peters, H. E. Koralewski, and E. Zerbst, "The principle of electrical carotid sinus nerve stimulation: a nerve pacemaker system for angina pectoris and hypertension therapy," Annals of Biomedical Engineering, vol. 8, no. 4-6, pp. 445-458, 1980.

[38] T. E. Lohmeier, E. D. Irwin, M. A. Rossing, D. J. Serdar, and R. S. Kieval, "Prolonged activation of the baroreflex produces sustained hypotension," Hypertension, vol. 43, no. 2, pp. 306$311,2004$.

[39] T. E. Lohmeier, T. M. Dwyer, E. D. Irwin, M. A. Rossing, and R. S. Kieval, "Prolonged activation of the baroreflex abolishes obesity-induced hypertension," Hypertension, vol. 49, no. 6, pp. 1307-1314, 2007.

[40] T. E. Lohmeier, T. M. Dwyer, D. A. Hildebrandt et al., "Influence of prolonged baroreflex activation on arterial pressure in angiotensin hypertension," Hypertension, vol. 46, no. 5, pp. 1194-1200, 2005.

[41] T. E. Lohmeier, D. A. Hildebrandt, T. M. Dwyer et al., "Renal denervation does not abolish sustained baroreflex-mediated reductions in arterial pressure," Hypertension, vol. 49, no. 2, pp. 373-379, 2007.

[42] J. Schmidli, H. Savolainen, F. Eckstein et al., "Acute devicebased blood pressure reduction: electrical activation of the carotid baroreflex in patients undergoing elective carotid surgery," Vascular, vol. 15, no. 2, pp. 63-69, 2007.

[43] J. A. Sloand, K. A. Illig, and J. D. Bisognano, "Improved control of resistant hypertension with device-mediated electrical carotid sinus baroreflex stimulation," Journal of Clinical Hypertension, vol. 9, no. 9, pp. 716-719, 2007.

[44] M. G. Mohaupt, J. Schmidli, and F. C. Luft, "Management of uncontrollable hypertension with a carotid sinus stimulation device," Hypertension, vol. 50, no. 5, pp. 825-828, 2007.

[45] D. A. Sica and T. E. Lohmeier, "Baroreflex activation for the treatment of hypertension: principles and practice," Expert Review of Medical Devices, vol. 3, no. 5, pp. 595-601, 2006.

[46] I. Scheffers, J. Schmidli, A. A. Kroon et al., "Sustained blood pressure reduction by baroreflex hypertension therapy with a chronically implanted system: 2-year data from the Rheos DEBUT-HT study in patients with resistant hypertension," Journal of Hypertension, vol. 26, supplement 1, p. S19, 2008.

[47] K. Heusser, J. Tank, S. Engeli et al., "Carotid baroreceptor stimulation, sympathetic activity, baroreflex function, and blood pressure in hypertensive patients," Hypertension, vol. 55, no. 3, pp. 619-626, 2010.

[48] K. Wustmann, J. P. Kucera, I. Scheffers et al., "Effects of chronic baroreceptor stimulation on the autonomic cardiovascular regulation in patients with drug-resistant arterial hypertension," Hypertension, vol. 54, no. 3, pp. 530-536, 2009.

[49] H. Karunaratne, S. Muluk, V. Papademetriou et al., "Implantation of a carotid baroreceptor stimulator in patients with pacemakers and hypertension," Pacing and Clinical Electrophysiology, vol. 34, no. 3, pp. 354-356, 2011.

[50] L. A. Sanchez, K. Illig, M. Levy et al., "Implantable carotid sinus stimulator for the treatment of resistant hypertension: local effects on carotid artery morphology," Annals of Vascular Surgery, vol. 24, no. 2, pp. 178-184, 2010. 


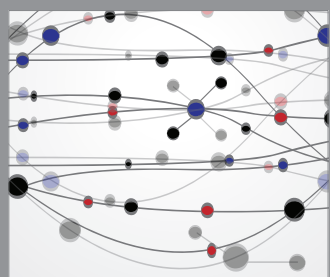

The Scientific World Journal
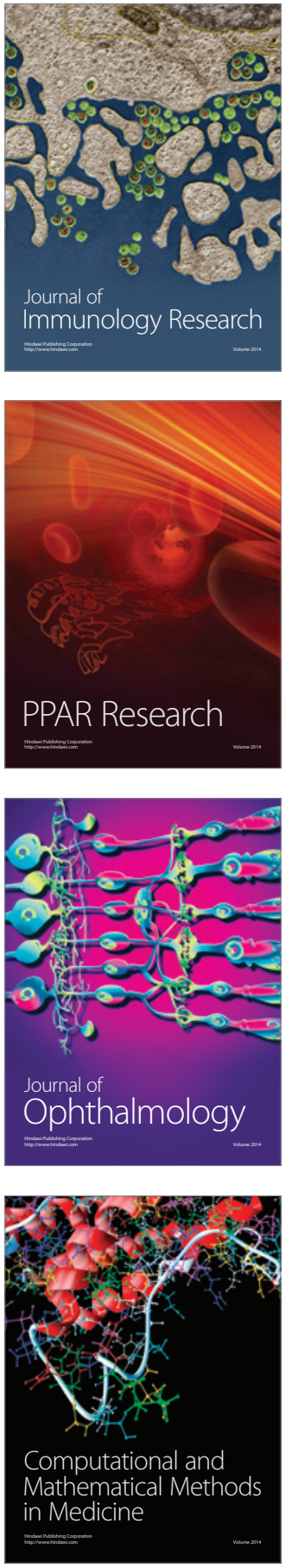

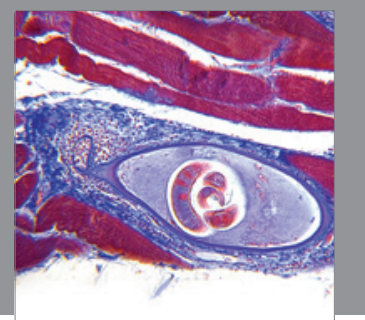

Gastroenterology

Research and Practice
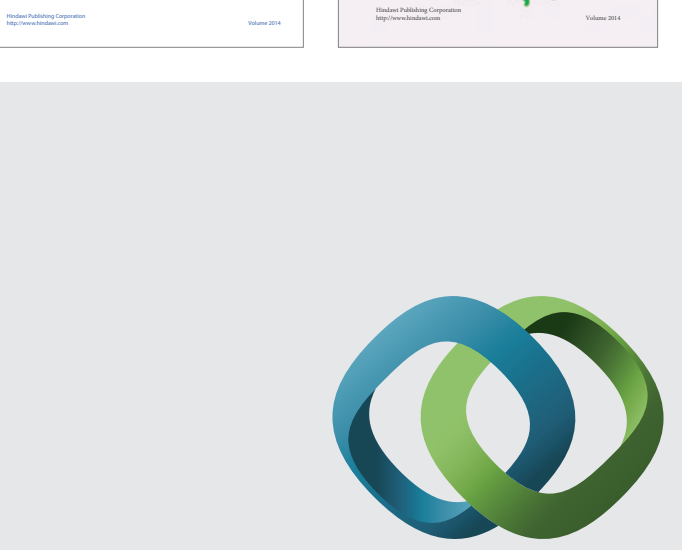

\section{Hindawi}

Submit your manuscripts at

http://www.hindawi.com
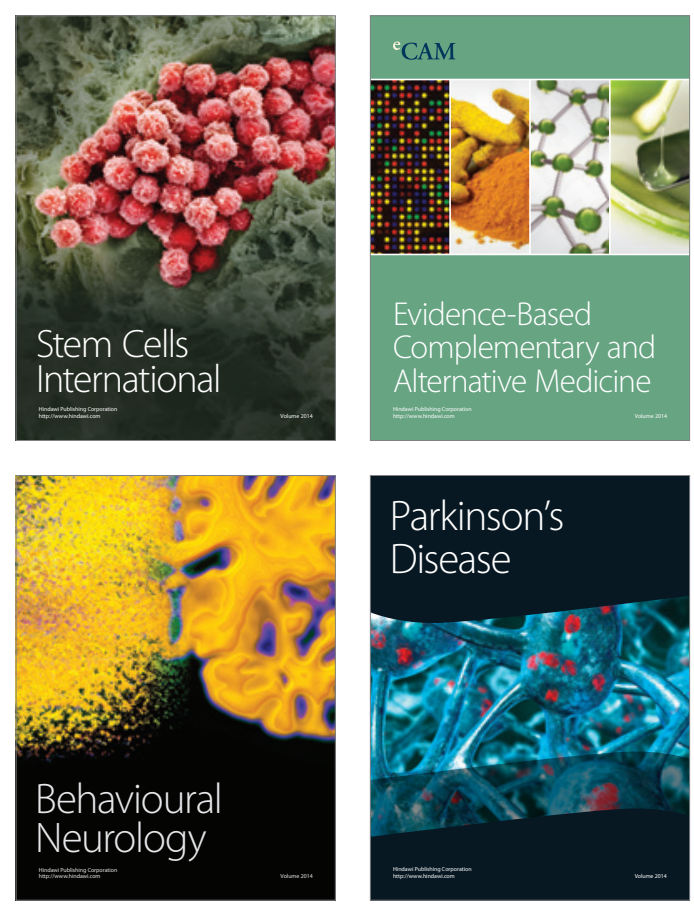

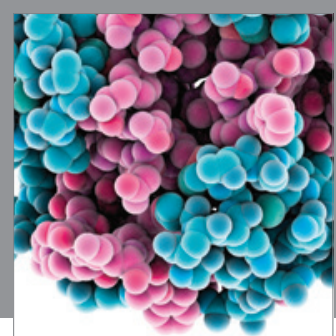

Journal of
Diabetes Research

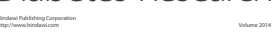

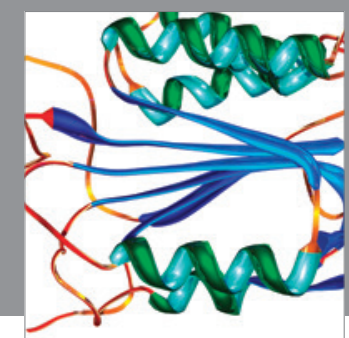

Disease Markers
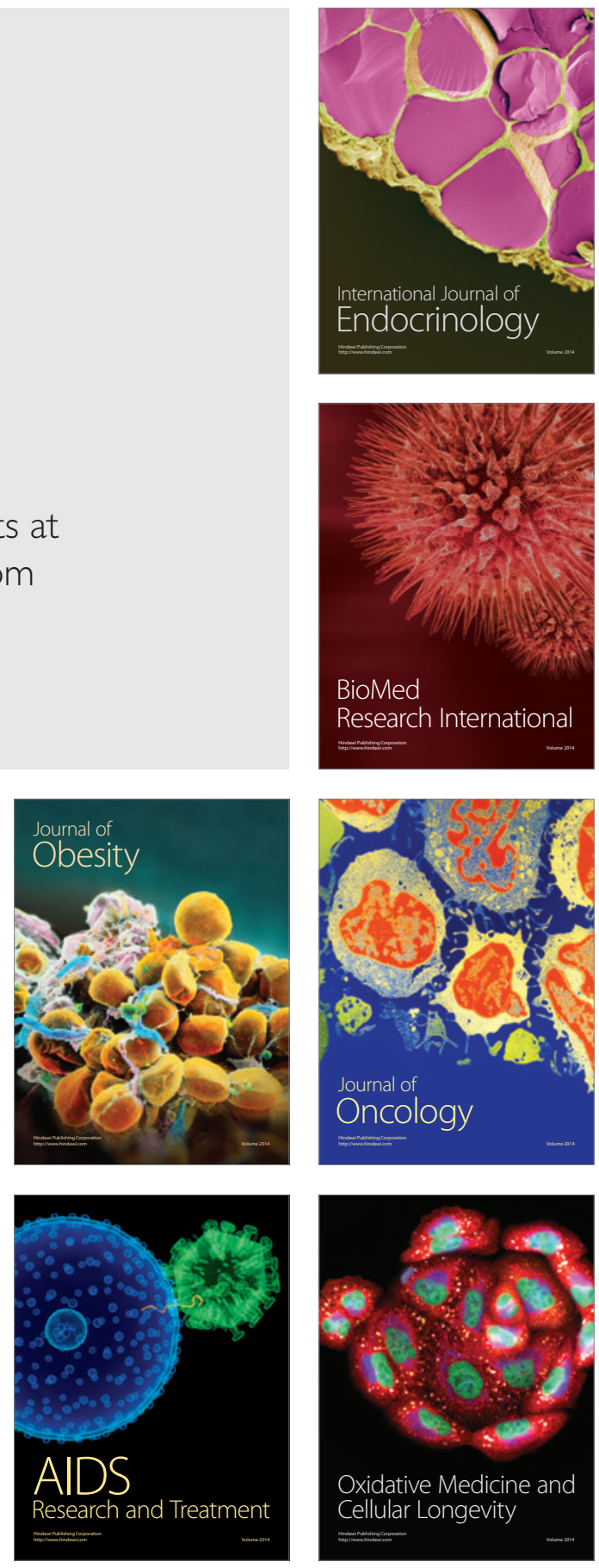